hailed from Uberaba. $37.6 \%$ had other concomitant STDs. $45.4 \%$ had their first appointment to specialised care done only on the second trimester. The patients had an average of 7 prenatal appointments and the majority (79.1\%) were using antiretroviral therapy (Biovir and Kaletra) during this period. On births, $1.4 \%$ ended in miscarriage; $26.0 \%$ were pre-term deliveries; $66.66 \%$ were on term deliveries; $1.44 \%$ were postterm deliveries and $4.3 \%$ were delivered elsewhere outside the HC-UFTM. Caesarean sections responded for $63.6 \%$ of the births and all the pregnant women received zidovudin (AZT) before their deliveries (+/-2.93 hour). On newborns, $63.76 \%$ had their weights between $2500 \mathrm{~g}$ and $4000 \mathrm{~g}$ and $92.6 \%$ had their APGAR $\geq 7$; all newborns received AZT after their births. Conclusion Obstetrical assistance to HIV-positive women is fundamental on their adherence to medication and on the reduction of vertical transmission. Those women must receive specialised care as soon as possible and the staff must be trained.

\section{P3.180 SYPHILIS AND PREGNANCY: DIRECTING PUBLIC HEALTH}

MC Paschoini, GC Montes, ALN Mendonça, JU Ribeiro, CCHB Oliveira. Universidade Federal do Triangulo Mineiro, Uberaba - MG, Brazil

\subsection{6/sextrans-2017-053264.415}

Introduction Measure the incidence of syphilis among pregnant women overseen by Hospital de Clínicas from Universidade Federal do Triângulo Mineiro. Outline their epidemiological profile and assess their obstetrical assistance and immediate neonatal data.

Methods Retrospective study from survey of medical records from January 2007 to December 2015 of pregnant women diagnosed with syphilis, followed by the application of a structured questionnaire evaluating their epidemiological, obstetrical and neonatal data.

Results There were 226 cases of pregnant women with syphilis. This number grew from 9 cases (3.9\%) in 2007 to 93 (41.2\%) in 2015. Patients were, on average, 23.6 years old, ranging from 10 to 45 years. On obstetrical data, 74 (33.6\%) were on their first pregnancy and the remaining had one or more pregnancies. $68.6 \%$ went to prenatal appointments and $69.4 \%$ were diagnosed on the second semester of their pregnancies. In $60.5 \%$ of the cases, the partner had not undertaken treatment. On births, $64.6 \%$ were vaginal births, $31.4 \%$ pre-term, $63.7 \%$ on term and $0.4 \%$ post-term births. On newborns, 68.6\% weighed between 2,000-4000 grams; $72.1 \%$ had $A P G A R \geq 7 \%$ and $31 \%$ were diagnosed with congenital syphilis and hospitalised for further treatment.

Conclusion Congenital syphilis remains a challenge to public health mainly due to the alarming rise in new infections on recent years. It was present on women of all ages amid their reproductive period. Syphilis deserves attention because of its impact on mothers, partners and newborns.

\section{P3.181 HEPATITIS C: CHALLENGING MODERN OBSTETRICS}

MC Paschoini, LN Resende, MM Mendonça, GPM Gomide, JU Ribeiro. Universidade Federal do Triangulo Mineiro, Uberaba - MG, Brazil
Introduction Asses the incidence of Hepatitis $\mathrm{C}$ on pregnant women overseen by the Hospital de Clínicas from Universidade Federal do Triângulo Mineiro; Delineate the Hepatitis Cinfected patients' epidemiological profile.

Methods Survey through Hospital de Clínicas records from 2007 to 2015 for Hepatitis C-infected pregnant women, followed by a review of medical records and by the administration of a structured questionnaire evaluating epidemiological, clinical and neonatal data.

Results 44 cases of Hepatitis C were found in the survey's period - an incidence of $0.4 \%$ on the births. On demography, the age of patients varied from 14 to 47 years, with an average of 30 years. The majority of infections were to non-white $(56.8 \%)$, single $(63.6 \%)$ and hailing from Uberaba $(81.8 \%)$ patients. The majority of surveyed women $(46.5 \%)$ have been diagnosed prior to their pregnancy. On obstetrical aspects, $61.3 \%$ had up to 2 previous pregnancies; $52.27 \%$ of the women visited the specialised sector during their second trimester; 2 patients had related clinical symptoms; $13.63 \%$ missed clinical appointments and 1 patient has been admitted without any prenatal consultation. Viral load was undetectable on 22 patients and 3 patients had a viral load $\geq 100000$ copies. On births, $84.2 \%$ of them were on term; $57.89 \%$ had vaginal births. Newborn's weight varied from 1725 to 4135 grams and $89.4 \%$ had Apgar $\geq 7$.

Conclusion Hepatitis $\mathrm{C}$ had a higher prevalence on pregnant women over 30 years old. The first specialised service appointment was mainly on the second trimester, probably due to delays on dispensation of public health services. Contagious and infectious diseases are a major challenge to public health, thus screening must be carried on to minimise vertical and horizontal transmissions.

\section{P3.182 OBSTETRIC ASSISTANCE TO HEPATITIS B-INFECTED WOMEN: STRATEGIES AND DIRECTIONS}

MC Paschoini, LN Resende, MM Mendonça, GPM Gomide, MCS Scandiuzzi. Universidade Federal do Triangulo Mineiro, Uberaba - MG, Brazil

\subsection{6/sextrans-2017-053264.417}

Introduction Measure the incidence of Hepatitis B (HB) among pregnant women overseen by Hospital de Clínicas from Universidade Federal do Triângulo Mineiro. Outline their epidemiological profile and asses their obstetric assistance.

Methods Survey of the records of pregnant women with a diagnosis of Hepatitis B from 2007 to 2015, medical record revision and application of a structured questionnaire evaluating their epidemiological, obstetrical and neonatal data.

Results There were 68 cases of Hepatitis B, that is, $0.6 \%$ of the births of the surveyed period. On demography, the mean age of the patients was 25.6 years, with variation from 16 to 43 years; the majority of the patients were white $(48.5 \%)$ and single $(45.58 \%)$. On obstetrical data, $54.4 \%$ had up to 2 previous pregnancies; $47.5 \%$ of their first appointments to specialised services were on the second semester; 2 patients had related medical symptoms and $10.29 \%$ missed any appointments. On their births, $88.13 \%$ were on term and $66.7 \%$ were vaginal births. Newborn weights were between 1035 and 4080 grams, $91.52 \%$ had $A P G A R \geq 7 \%$ and $88.1 \%$ received 